\title{
Antibacterial effect of lidocaine in various clinical conditions
}

Received April 10, 2018

Revised 1st, August 18, 2018

2nd, October 8, 2018

Accepted October 23, 2018

\section{Corresponding author}

Young Duck Shin, M.D.

Department of Anesthesiology and Pain Medicine, Chungbuk National University Hospital, Chungbuk National University College of Medicine, 776, 1sunhwan-ro, Seowon-gu, Cheongju 28644, Korea Tel: 82-43-269-6234

Fax: 82-43-272-0264

E-mail: yydshin@naver.com ORCID

https://orcid.org/0000-0002-8373-8478

\section{Hyeon Tae Kim, Seung-Woon Lim, Kyoung Hoon Yim, Sang Hi Park, Jung Hee Choi, Yoo-Mee Bae, Il Dong Shin, and Young Duck Shin}

Department of Anesthesiology and Pain Medicine, Chungbuk National University Hospital, Chungbuk National University College of Medicine, Cheongju, Korea

Background: Infection, one of the complications associated with procedures, can cause fatal outcomes for patients. Although the local anesthetic agent we use is less susceptible to infection due to its antibacterial action, we performed this study to check the change in the antibacterial effect of lidocaine in various clinical conditions.

Methods: After exposing lidocaine to five contaminated environments, we checked on whether the bacteria could be cultured in blood agar plate (BAP) media. In each contaminated environment, lidocaine was exposed for $4 \mathrm{~h}(\mathrm{n}=9)$ and $8 \mathrm{~h}(\mathrm{n}=9)$, and the results were compared. Lidocaine was swabbed with chlorhexidine (group $A$ ), brought into contact with saliva (group B), skin (group C), an operating room floor and an outpatient room floor (group D), operating room air for $24 \mathrm{~h}$ (group A-a), and outpatient room air for $24 \mathrm{~h}$ (group A-b). After exposure, the culture was initiated.

Results: In 2 of 9 BAP media where lidocaine was exposed to saliva (group B) for $8 \mathrm{~h}$, growth of a colony was observed. In gram staining, it was found to be Streptococcus viridans. No bacteria were found in any other groups.

Conclusions: Though lidocaine has strong antibacterial activity, it has been found that long-term exposure to a contaminated environment reduces its antibacterial activity and that drug contamination can be heavily affected not only by environmental but also human effects. Therefore, the use of aseptic drugs is necessary, and stopping the reuse of the drug is a way to prevent complications, including infection.

Keywords: Drug contamination; Lidocaine.

\section{INTRODUCTION}

Local anesthetics, such as lidocaine or levobupivacaine, which we use in our procedures, have their own antibacterial effects [1], and their antibacterial effects have already been identified in previous studies [2]. All of our procedures can cause various complications, such as infection, bleeding, dura puncture, and post intervention pain. Though there is no report on overall incidence of infection, which is one of the complications, it is reported that the incidence rate of in- fectious spondylodiscitis after spine surgery is $0.05-5.3 \%$ [3]. And in very rare cases, there has been a psoas abscess after injection at the pain trigger point of the spinal muscle [4]. Based on these findings, although infection is not common, it is an important complication to be avoided because of the variety of sites and features. Causes of infection include air contamination, carelessness of the operator, blood circulation of the primary infection, and the most common cause during the procedure is contamination of the needle entry path due to patient skin contamination. Though local anesthetics we

This is an Open Access article distributed under the terms of the Creative Commons Attribution Non-Commercial License (http://creativecommons.org/licenses/by-nc/4.0) which permits unrestricted non-commercial use, distribution, and reproduction in any medium, provided the original work is properly cited. 
use are believed to be relatively safe for infection due to their antibacterial effect, little is known about their degree of antibacterial effect. Thus, we started this study to detect hospital pathogens by exposing the drug to various contaminated environments and checking how much we should be careful and prepared as we perform surgery.

\section{MATERIALS AND METHODS}

The author carried out experiments to identify contamination of lidocaine in five contaminated environments. A preservative free $1 \%$ lidocaine, manufactured at the same time with an expiry date of October 2019 was used (Lidocaine hydrochloride, Daihan Pharm, Korea).

The lidocaine, employed for the present study, was divided into 5 groups (A-E). And for each group, the times of the local anesthetics to be exposed to a common contaminated environment were set $4 \mathrm{~h}$ and $8 \mathrm{~h}$ respectively.

Group A was comprised of 36 specimens of the lidocaine. Then, group A was divided into group A-a (18 specimens) and group A-b (18 specimens). In what follows, groups B, C, D, and $E$ were also assigned with 18 specimens of lidocaine.

Group A which were swabbed with chlorhexidine; the samples collected thereby were then cultured. The cultured samples were then divided into specimens for group A-a and group A-b, wherein the group A-a was assigned with the 18 specimens which were preserved for $24 \mathrm{~h}$ in the operating room. Likewise, the group A-b was assigned with 18 specimens preserved for $24 \mathrm{~h}$ in an outside setting to distinguish the potential differences in an airborne contamination which could occur in the course of common handling between an operating room and an outside setting (Fig. 1). In this case, the 18 bottles of lidocaine specimen, assigned to group B, were put into an experiment after they were swabbed with saliva; the setting intended for the simulation of vials exposed to saliva sputtered from a conversation among common clinical staff workers who process vials without wearing masks. Likewise, there were 18 specimens of lidocaine, assigned to group C, which were contacted with the unclean skin of the operator to simulate the situations of handling vials with unsterile hands, or the case of how the vials could come to be in common contact with the skin of operators. The 18 specimens, equally assigned to group D and group E respectively, were employed to simulate the situations of vials fallen to the labo- ratory floors in the operating room and in an outdoor setting (Fig. 2).

The number of specimens in each group, 18, was determined by taking the period for preservation of specimens before culture, the number of possible treatments for each day in our department, and the availability of the intramural laboratory for the culture into account.

In terms of collecting lidocaine in the operating room, a lidocaine vial lid was opened during preparation for the procedure. About $10 \mathrm{~min}$ later, the vial was gripped with ungloved hands, and the drug was collected in a $10 \mathrm{ml}$ syringe (Piltech Corp., Korea) with gloved hands. A syringe with a preinstalled needle was used. Drug preparation was performed on a table about $1 \mathrm{~m}$ away from the procedure bed. As the procedure proceeded, the operating room's doors were opened and closed about 3 times on average, about 30 times on average over $24 \mathrm{~h}$. An average of 5 people, including the patient, participated in the procedure. In the outpatient room, the drug was prepared in a process identical to that in the operating room. It was prepared on a table between two beds used for outpatient procedures, and this table was located about $50 \mathrm{~cm}$ from the bed. For samples collected in the operating room and outpatient room, $0.2 \mathrm{ml}$ per sample was spread on a blood agar plate (BAP) (Asan Pharm, Korea) using the streak plate method at a clean bench (laminar flow hood) in a microbial culture room. It was incubated for $72 \mathrm{~h}$ in an

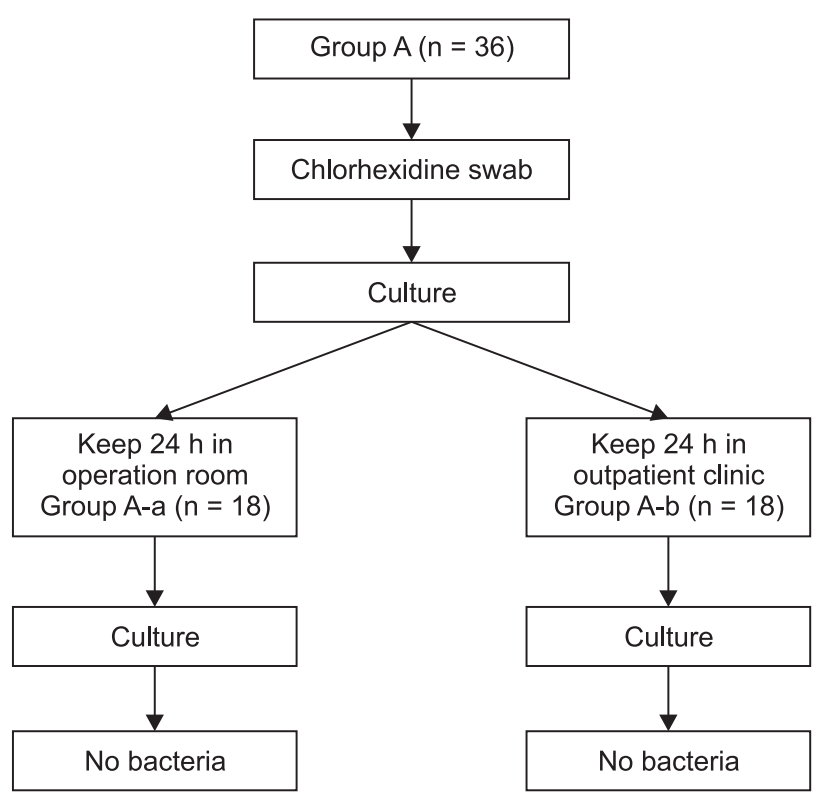

Fig. 1. A diagram of an experimental procedure for group A. 


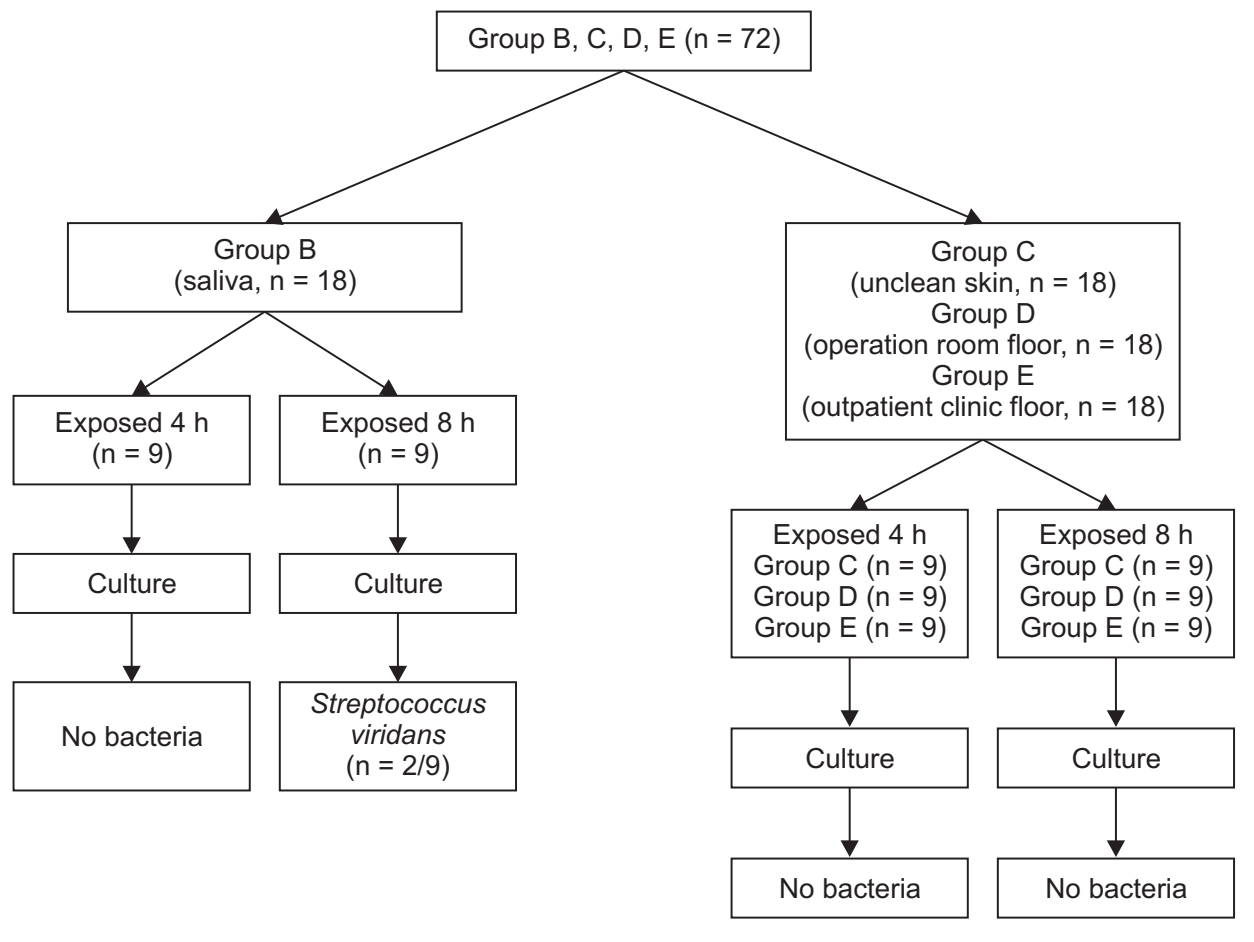

Fig. 2. A diagram of an experimental procedure for group B-E.

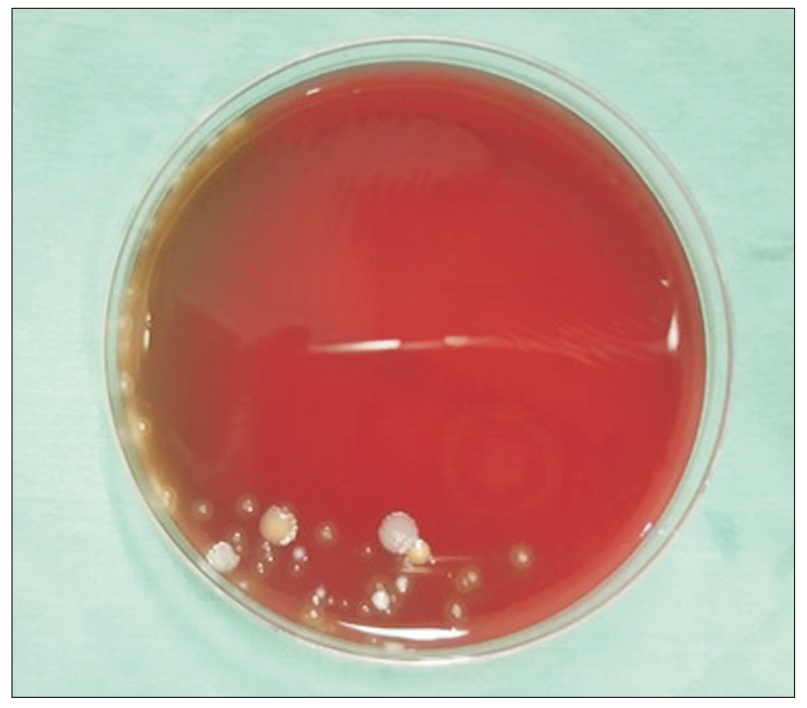

Fig. 3. Image of blood agar plate medium which culture sample exposed to saliva. Colony is observed at the bottom of the plate.

incubator (VS-2180C-CO2 incubator, Vision science, Korea), and identification of the bacteria was determined to be done when there was a colony growth in BAP medium as a result of culture.

\section{RESULTS}

The result of the culture of lidocaine in BAP showed that

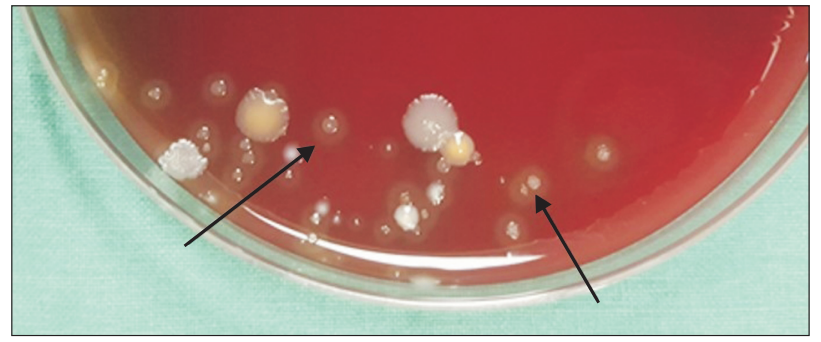

Fig. 4. Close-up image of blood agar plate medium which culture sample exposed to saliva. Hemolysis around the colony is observed as the bacteria grows in the plate (arrows).

the bacterium grew in group $B$ and that it was identified to be Streptococcus viridans in gram staining (Figs. 3, 4). Bacteria did not grow in the remaining groups, and there was no sign of foreign matter or contamination inside the vial (Fig. 5, Table 1).

\section{DISCUSSION}

There are many studies on the inhibition effect of local anesthetics, such as bupivacaine, ropivacaine, lidocaine, and L-bupivacaine, on bacterial growth. Among these, lidocaine specifically inhibits growth of bacteria by affecting cell walls and cytoplasmic membranes [5]. It also inhibits membrane-bound enzymes and inhibits RNA or DNA that 
synthesize proteins [6]. There is also a study in a rat model in which continuous lidocaine infusion reduces Streptococcus aureus-induced infections, thus local anesthetics can prevent surgical wound infection [7]. There are various types and concentrations of local anesthetics used in a clinic. Commonly used bupivacaine $0.125-0.75 \%$, lidocaine $1-3 \%$, inhibits growth of bacteria and fungi in various conditions. High concentrations, long exposure times, and high temperatures are correlated with a proportional increase in microbial inhibition, respectively [1]. Also, the addition of other agents to preservatives, intravenous anesthetics such as opioids or propofol modifies antibacterial activity through synergistic or antagonistic action. Therefore, the antibacterial effect of local anesthetics can't be unconditionally trusted [1]. This is why we should always think of complications like infections when we perform procedures.

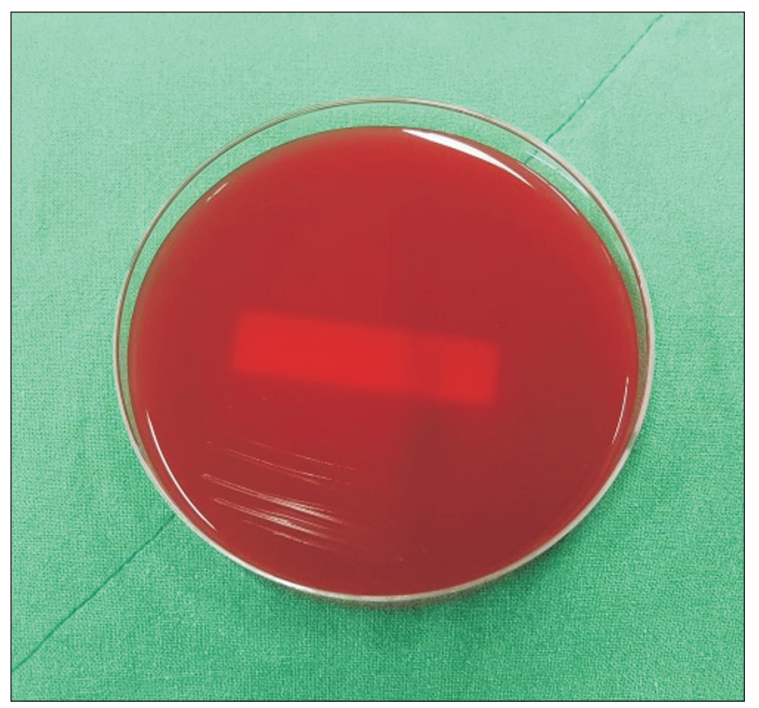

Fig. 5. Close-up image of blood agar plate medium without bacteria growth. Smear marks at the bottom of the badge. No specific colony and hemolysis, sign of bacterial growth, was seen.
In this experiment, we exposed lidocaine to various contaminated conditions. Lidocaine was used because no preservatives were added to it, because it was used frequently during procedures, and because it was more likely to be repeatedly used because of its vial formulation. Since preservatives are not added, it should be used within 36 months of manufacture and should be used as soon as possible after opening.

As aforementioned, there have been studies in the literature review which delved into the antimicrobial activity of local anesthetics including lidocaine; most of such studies, however, had been carried out in a limited environment of laboratories. Recently, the cases of infection of patients owing to contaminated drugs or infusions have been reported frequently from private- and general hospitals to date. This brings us to understand that long period of times and many efforts on the part of the patients are frequently needed to restore damages of patients which have resulted from the misuse of contaminated drugs. The present study was thus conceived by the necessity to identify the degree of antimicrobial activity of the local anesthetics through the experimental schemes designed to simulate potential environment of contamination in spaces, providing patients with pertinent treatment or operation, and to remind the operators to be aware of the potential consequences which can be the result from contact with a contaminated environment.

The authors of the present study have assumed that, the local anesthetics, exposed to environmental and human effects, could in fact be contaminated beyond the level of the function of their spontaneous antimicrobial activities.

However, study result suggest that the antibacterial effect of local anesthetics is stronger than expected, as bacteria did not grow in most of extreme environments. This suggests that human factors may play a much more important role than air

Table 1. Result of Samples Cultured in BAP Medium which were Exposed to Contaminated Environments

\begin{tabular}{|c|c|c|c|c|c|c|c|}
\hline Variables & $\begin{array}{l}\text { Group A } \\
(n=18)\end{array}$ & $\begin{array}{l}\text { Group B } \\
(n=18)\end{array}$ & $\begin{array}{l}\text { Group C } \\
(n=18)\end{array}$ & $\begin{array}{l}\text { Group D } \\
(n=18)\end{array}$ & $\begin{array}{l}\text { Group E } \\
(n=18)\end{array}$ & $\begin{array}{c}\text { Group A-a } \\
(n=18)\end{array}$ & $\begin{array}{c}\text { Group A-b } \\
(n=18)\end{array}$ \\
\hline 4-h exposure $(n=9)$ & $\bigcirc$ & $\times$ & $\times$ & $\times$ & $\times$ & $\times$ & $\times$ \\
\hline 8-h exposure $(n=9)$ & $\times$ & $\bigcirc(n=2)$ & $x$ & $x$ & $\times$ & $\times$ & $x$ \\
\hline Bacterial identification & & $\begin{array}{c}\text { Streptococcus } \\
\text { viridans }\end{array}$ & & & & & \\
\hline
\end{tabular}

Group A: chlorhexidine swab, Group A-a: keep 24 h in operation room, Group A-b: keep 24 h in outpatient clinic, Group B: contact saliva, Group C: contact unclean skin, Group D: contact operation room floor, Group E: contact outpatient clinic floor. $\bigcirc$ : bacteria grew in culture result, $\times$ : bacteria didn't grow in culture result, $\mathrm{n}$ : sample number of lidocaine. 
pollution, operating room, and outpatient room, which are conventionally thought to be factors for the contamination. Results in groups exposed to saliva showed that bacteria did not grow in lidocaine that was exposed for $4 \mathrm{~h}$ but grew in lidocaine that was exposed for $8 \mathrm{~h}$, which indicates that longterm exposure to the contaminated environment weakens the antibacterial activity of the local anesthetic. In addition, it was confirmed that as the contamination level gets higher or the contamination is prolonged, the agent can be contaminated, losing its antibacterial activity. However, the result that no bacterial growth was seen in groups excluding the saliva-exposed group and groups exposed to a contaminated environment in the short term, does not mean the procedure environment and process are free from contamination. Since infection from contamination can occur with one or two of these factors, care should be always be taken not to contaminate the procedure process, even if the local anesthetic has antibacterial activity. In the cases of lidocaine or levobupivacaine, which are often used in procedures, there is no special preservative for long-term preservation of the opened drug. A small amount of additive, such as hydrochloric acid, added to the drug will only prevent the deterioration of the product for a short period of time during the procedure after the drug is opened. Therefore, avoiding drug reuse and prolonged use after opening and sterilizing vials, even if there was no difference in the result, will be the most efficient way to reduce drug-related infections in patients. Also, as the possibility of contamination increases when many people move during the procedure, both in the outpatient and the operating room, it is necessary to reduce the presence of floating dust and bacteria in the air by limiting the movement of people.

Infection accounts for $0.5 \%$ to $11 \%$ of complications in the operating room $[8,9]$. There are many causes of infection from sources such as airborne dust or bacteria of 5-7 $\mu \mathrm{m}$ in the air [10-12], contamination caused by the operator [13], and transmission of infection of primary sources including respiratory or urinary system [14], and so on. Among those, contamination of the needle entry path due to contamination of the patient's skin, which occurs during injection or the placing of a catheter is the most common cause [13]. There are many types and prevalence rates of infections resulting from these causes during the procedure and anesthesia. Epidural abscess, though rare, is an emergency state, with a prevalence rate of $0.2-1.2$ cases per 10,000 hospital inpatients per year in the United States (US) [14]. Bacterial meningitis is the most common central nervous system (CNS) infection, with an incidence rate of 2.5 cases/100,000 in the US [15]. In addition, the prevalence of infectious spondylodiscitis after spinal procedures is known to be $0.05-5.3 \%$ [3]. Perineural catheter infection has a prevalence rate of $0-3 \%$ [16].

Patient infection due to the contamination of medicines used in procedures is likely. In a report of infection outbreak resulting from the use of contaminated drugs, exserohilum rostatum fungal meningitis following epidural injection of contaminated methylprednisolone has been reported in several US since the end of September 2012. Methylprednisolone suspected of contamination at this time was 17,675 vials, and 751 of the 13,534 patients treated were infected. Of these, 233 had meningitis, 7 had stroke, 325 had spinal or paraspinal infection, 35 had osteoarticular infection, and 64 of the infected patients died [17]. Secondly, in Korea, there is a report of joint and cutaneous infection outbreak by nontuberculous mycobacteria after corticosteroid injection in 61 patients. In this case, infection outbreak resulted from the use of contaminated triamcinolone [18]. The above two examples show that the use of contaminated drugs can cause infection outbreaks.

Next, there is a report of an infection outbreak due to carelessness during procedures. In Korea, there was a report of a cutaneous infection outbreak caused by mycobacterium in 40 patients who had acupuncture in oriental medicine clinics. In this report, contamination of acupuncture needles or contamination of patients' skin are explained to be the cause of the infections [19]. This report is a representative example of how carelessness and improper disinfection of the practitioner can cause infection to the patient.

Infection after the procedure can be local or systemic, and many symptoms can manifest, such as fever, severe headache, low consciousness, severe back pain, root pain, or weakness. If the diagnosis is delayed, these symptoms can develop into complications, such as a CNS infection, that can cause fatal outcomes to patients, including death. Therefore, neurological examination, magnetic resonance imaginglike imaging, and blood tests should be performed promptly. Positive results, such as quick symptom improvement, good prognosis, and reduction of medical expenses, are expected when treatment by an infection specialist, antibiotic treatment, and surgical treatment are appropriately performed 
[15].

There is controversy as to which sterilization method is good for the procedure, but chlorhexidine and iodine are most commonly used. Although there may be differences depending on the situation and location of disinfection, chlorhexidine has recently been shown to work faster and more effectively than iodine, especially in the case of catheter placement [15]. And in the case of patients with short-term catheter placement, some studies have shown that skin disinfection with chlorhexidine-alcohol reduces catheter-induced infections more than iodine-alcohol disinfection [20]. According to the study by Daurouiche et al. [21], chlorhexidinealcohol has a better effect on both superficial incisional infection (4.2\% vs. $8.6 \%$ ) and deep incisional infection ( $1 \%$ vs. $3 \%$ ) than povidone-iodine, though there was no big difference in organ space infection ( $4.4 \%$ vs. $4.5 \%$ ) [5]. This suggests that the type and disinfection site of the disinfection drug may also affect the infection, but it is also important to perform disinfection properly.

As noted above, the local anesthetic itself has a strong antibacterial effect, but its effect decreases if exposed to a contaminated environment for a long time. It is also dangerous to unconditionally trust the antibacterial effect of local anesthetics, because it is likely to be contaminated by the environment and can be heavily impacted by human factors. It is important to prevent the reuse of local anesthetics in clinical settings and to use the drug in aseptic conditions and processes at all times in order to reduce the risk of infection, since infection may cause critical results to the patient.

The number of specimens identified in each group defined for the experiment conducted in the present study was considered to be insufficient, and there was only one group that manifested the cultured microorganisms from the dichotomized settings of $4 \mathrm{~h}$ and $8 \mathrm{~h}$, for the specimens to be exposed to a contaminated environment. Thereby, the unavailability of a sufficient statistical analysis due to fewer numbers in frequency of derived cases that disabled the buildup of control group, would be the biggest limitation noted in the present study. Future studies are thus suggested to be designed to attain proper statistical causalities through increasing the number of samples and reducing the experimental environment with the establishment of a formal control group, to derive significant results capable of reducing the risk of the intramural infection of patients.

\section{ACKNOWLEDGMENTS}

This work was supported by Chungbuk National University Hospital grant in 2018.

\section{CONFLICTS OF INTEREST}

No potential conflict of interest relevant to this article was reported.

\section{ORCID}

Hyeon Tae Kim: https://orcid.org/0000-0002-3581-9767

Seung-Woon Lim: https://orcid.org/0000-0002-9720-4055

Kyoung Hoon Yim: https://orcid.org/0000-0001-9293-3833

Sang Hi Park: https://orcid.org/0000-0003-1138-6028

Jung Hee Choi: https://orcid.org/0000-0002-4142-6505

Yoo-Mee Bae: https://orcid.org/0000-0003-1954-4828

Il Dong Shin: https://orcid.org/0000-0002-1426-5355

\section{REFERENCES}

1. Johnson SM, Saint John BE, Dine AP. Local anesthetics as antimicrobial agents: a review. Surg Infect (Larchmt) 2008; 9: 205-13.

2. Adler DMT, Damborg P, Verwilghen DR. The antimicrobial activity of bupivacaine, lidocaine and mepivacaine against equine pathogens: an investigation of 40 bacterial isolates. Vet J 2017; 223: 27-31.

3. Lee SJ, Choi EJ, Nahm FS. Spondylodiscitis after cervical nucleoplasty without any abnormal laboratory findings. Korean J Pain 2013; 26: 181-5.

4. Kim DH, Kim HS. Epidural and psoas abscesses recognized after paravertebral trigger point injection: a case report. Korean J Pain 2007; 20: 74-7.

5. Begec Z, Gulhas N, Toprak HI, Yetkin G, Kuzucu C, Ersoy MO. Comparison of the antibacterial activity of lidocaine $1 \%$ versus alkalinized lidocaine in vitro. Curr Ther Res Clin Exp 2007; 68: 242-8.

6. Liu K, Ye L, Sun W, Hao L, Luo Y, Chen J. Does use of lidocaine affect culture of synovial fluid obtained to diagnose periprosthetic joint infection (PJI)? Med Sci Monit 2018; 24: 448-52.

7. Lu CW, Lin TY, Shieh JS, Wang MJ, Chiu KM. Antimicrobial effect of continuous lidocaine infusion in a Staphylococcus aureus-induced wound infection in a mouse model. Ann Plast Surg 2014; 73: 598-601.

8. National Nosocomial Infections Surveillance System. National 
Nosocomial Infections Surveillance (NNIS) System Report, data summary from January 1992 through June 2004, issued October 2004. Am J Infect Control 2004; 32: 470-85.

9. Klevens RM, Edwards JR, Richards CL Jr, Horan TC, Gaynes RP, Pollock DA, et al. Estimating health care-associated infections and deaths in U.S. hospitals, 2002. Public Health Rep 2007; 122: 160-6.

10. Gosden PE, MacGowan AP, Bannister GC. Importance of air quality and related factors in the prevention of infection in orthopaedic implant surgery. J Hosp Infect 1998; 39: 173-80.

11. Whyte W, Hodgson R, Tinkler J. The importance of airborne bacterial contamination of wounds. J Hosp Infect 1982; 3: 123-35.

12. Dharan S, Pittet D. Environmental controls in operating theatres. J Hosp Infect 2002; 51: 79-84.

13. Reynolds F. Infection as a complication of neuraxial blockade. Int J Obstet Anesth 2005; 14: 183-8.

14. Grewal S, Hocking G, Wildsmith JA. Epidural abscesses. Br J Anaesth 2006; 96: 292-302.

15. Hebl JR, Niesen AD. Infectious complications of regional anesthesia. Curr Opin Anaesthesiol 2011; 24: 573-80.

16. Nicolotti D, Iotti E, Fanelli G, Compagnone C. Perineural catheter infection: a systematic review of the literature. J Clin Anesth
2016; 35: 123-8.

17. Kaufman CA, Malani AN. Fungal infections associated with contaminated steroid injections. In: Emerging infections 10. Edited by Scheld WM, Hughes JM, Whitley RJ. Washington, DC, ASM Press. 2016, pp 359-74.

18. Jung SY, Kim BG, Kwon D, Park JH, Youn SK, Jeon S, et al. An outbreak of joint and cutaneous infections caused by non-tuberculous mycobacteria after corticosteroid injection. Int J Infect Dis 2015; 36: 62-9.

19. Song JY, Sohn JW, Jeong HW, Cheong HJ, Kim WJ, Kim MJ. An outbreak of post-acupuncture cutaneous infection due to Mycobacterium abscessus. BMC Infect Dis 2006; $6: 6$.

20. Mimoz O, Lucet JC, Kerforne T, Pascal J, Souweine B, Goudet V, et al. Skin antisepsis with chlorhexidine-alcohol versus povidone iodine-alcohol, with and without skin scrubbing, for prevention of intravascular-catheter-related infection (CLEAN): an openlabel, multicentre, randomised, controlled, two-by-two factorial trial. Lancet 2015; 386: 2069-77.

21. Darouiche RO, Wall MJ Jr, Itani KM, Otterson MF, Webb AL, Carrick $\mathrm{MM}$, et al. Chlorhexidine-alcohol versus povidone-iodine for surgical-site antisepsis. N Engl J Med 2010; 362: 18-26. 